\title{
Hydrogen at megabar pressures and the importance of ortho-para concentration
}

\author{
Isaac F Silvera and Michael G Pravica \\ Lyman Laboratory of Physics, Harvard University, Cambridge, MA 02138, USA
}

Received 1 June 1998

\begin{abstract}
Hydrogen and its isotopes are discussed, showing the known phase diagrams at high pressures, with emphasis on efforts to observe the insulator-metal transitions. The role that the ortho-para concentration has played in the challenging effort to produce metallic hydrogen is discussed, showing recent NMR results on the determination of the conversion rate constant, important for knowing the state of a sample at high pressure.
\end{abstract}

\section{Introduction}

One of the important challenges of this century has been to transform hydrogen to the metallic state by compressing it to very high densities. This problem was first set forth by Wigner and Huntington [1] who proposed that at a sufficiently high density the molecules at the lattice sites would dissociate to form an atomic lattice with half filled bands so that the solid would be metallic. In the more than half a century since this prediction the expected pressure has risen from 0.25 megabar to the 3-6 megabar (300-600 GPa) region as experimentalists have probed to ever higher pressures and theorists have refined the calculational techniques. The prediction in 1968 by Ashcroft [2] that metallic hydrogen might be a high-temperature, perhaps room temperature superconductor, spurred on the experimental developments to produce this material. Subsequently, it was realized by Ramaker et al [3] that even in the molecular state, hydrogen might become metallic by a band overlap mechanism. In recent years this mechanism has been pursued by a large number of researchers [4] and the prediction of the metallization pressure in this form has been as low as $150 \mathrm{GPa}$. Recent calculations predict that molecular metallic hydrogen might have a substantially higher critical temperature for superconductivity than does the atomic form [5]. Measurements on physical properties of hydrogen have been carried out to pressures as high as $251 \mathrm{GPa}[6,7]$ and hydrogen has been pressurized to $342 \mathrm{GPa}$ [8], but the samples remain transparent and there is as yet no indication of metallization. Recent measurements [9] of the equation of state of molecular hydrogen to pressures somewhat over a megabar have been used to calculate the Gibbs free energy. This can then be extrapolated and compared to theoretical equations of state for the metallic phase. When these curves cross, a transition takes place from one phase to the other. The prediction here is around $600 \mathrm{GPa}$. The current status is that theory has not been good enough to accurately predict any of the metallization transitions. Experiment has gradually raised the bar, aiming at a level which seems to rise higher and higher as the bar goes up. It seems that the bar and the level will eventually cross. But for it to happen in this century the pace must pick up. 
Metallic hydrogen itself will present an exciting area of research, especially if it can be reproduced and carried out in table-top experiments under static conditions in many laboratories. Here we cite a few of the areas and issues as questions-without labouring to provide answers. What is the form of the $P-T$ phase line for the metal-insulator transition? How does such a line meet the liquid phase? What is the order of the phase transition? Do new phases exist at still higher pressures? What is the isotope effect on the transition, found by studying hydrogen and deuterium? What role does the zero-point motion play in the transition temperature? How do impurities affect the transition? Is atomic metallic hydrogen a high temperature superconductor? Is molecular metallic hydrogen a high temperature superconductor? Is the transition to the atomic phase metastable? Can metallic hydrogen be produced and stabilized at ambient pressure? Is metallic hydrogen useful as a propellant? Can atomic hydrogen or deuterium be produced in a liquid state at zero Kelvin due to the large zero-point motion? Clearly this is a fascinating list of questions, awaiting the metallization of hydrogen.

Why has theory had difficulty in predicting the metallization of hydrogen? The main approach has been to calculate the free energy of hydrogen in the molecular phase and the metallic phase. When the free energy curves cross, the transition takes place. The free energy is dominated by terms which are best calculated by techniques of electronic structure of solids, in particular the density functional approach. These methods give accurate results if the lattice is rigid without zero-point motion, so that a so-called ball-and-stick model of molecules can be used. Calculations are for rigid static molecules situated at lattice sites and take into account the distribution of electronic clouds about the atomic centres. In reality the molecules are in rotational-vibrational states. The extreme example occurs at low pressure and temperature where the molecular distributions can be described by spherical harmonics $Y_{J M}(\Omega)$ with $J$ and $M$ the rotational quantum numbers and $\Omega$ the set of angles which describe the orientation of the molecular axes with respect to a reference frame. In the $J=0$ state, the dumbbell shaped molecules have a spherical distribution. But there is more: the protons in a given molecule have a large relative zero-point motion and the molecules themselves have a large correlated zero-point motion about their lattice sites. Conventional electronic band structure calculations cannot handle the rotational or translational zero-point motions which are a fundamental and significant part of the manybody energy of these light solids. Ashcroft [10] has shown that the orientational states of the molecules have an important effect on the structure, while Garcia et al [11] have shown that there is a large difference in the critical pressure for a transition depending on whether the molecules are in spherical or oriented states.

\section{The experimental phase diagrams at high pressure}

The phase diagrams of hydrogen and its isotopes in the solid state have been studied to about $200 \mathrm{GPa}$ and from liquid helium temperature to room temperature. To clearly classify the phase diagrams we start by discussing the ortho-para species of hydrogen.

Hydrogen has two species, ortho and para. This arises from the indistinguishability of the protons and the Pauli principle. Since the total wavefunction of an isolated molecule must be antisymmetric under the exchange of the two spin $1 / 2$ protons, then the antisymmetric total nuclear spin state of a molecule, $I=i_{1}+i_{2}=0$, must couple with rotational states which are symmetric under permutation of the nuclei; these states are called para. The symmetric spin states $I=1$ couple with the antisymmetric orientational states and are called ortho. By considering low pressure and temperature, we have an example of the extreme differences of the para and ortho species. Under these conditions the molecules in the solid behave 
to a large extent as isolated molecules with weak anisotropic intermolecular interactions, with the imposition of the symmetry requirements of the solid. As mentioned above, the single molecule orientational states are the free-rotor spherical harmonics. The para species are in the symmetric spherical harmonic states $Y_{00}$ and the ortho are in the antisymmetric states $Y_{1 M}$. The former are spherically symmetric while the latter are figure-eight shaped $\mathrm{p}$ wavefunctions, and have anisotropic distributions in space. Pure para, whose molecules have ball-like distributions, remains hexagonal close packed (hcp) down to $T=0 \mathrm{~K}$. The weak electric quadrupole-quadrupole (EQQ) interactions average to zero in this state. However, the anisotropic forces between molecules increase with density, so that at very high pressure, even the para lattice transforms into an orientationally ordered state. This is called the BSP (broken symmetry phase) transition, as the symmetry of the ground molecular wavefunctions is broken. The spherical harmonics are no longer single molecule eigenstates and the new ground state has anisotropic, but symmetric (as required for para species) orientational wavefunctions. An example is given in figure 1, the phase diagram for deuterium (for deuterium the $J=0$ solid corresponds to ortho); here we see the LP (low pressure) hcp phase transforms to the BSP at $28 \mathrm{GPa}$. At about $160 \mathrm{GPa}$ the deuterium A (D-A) phase appears. A similar phase diagram exists for hydrogen (with the H-A at about $150 \mathrm{GPa}[12]$ and the BSP at $110 \mathrm{GPa}$ [13]).

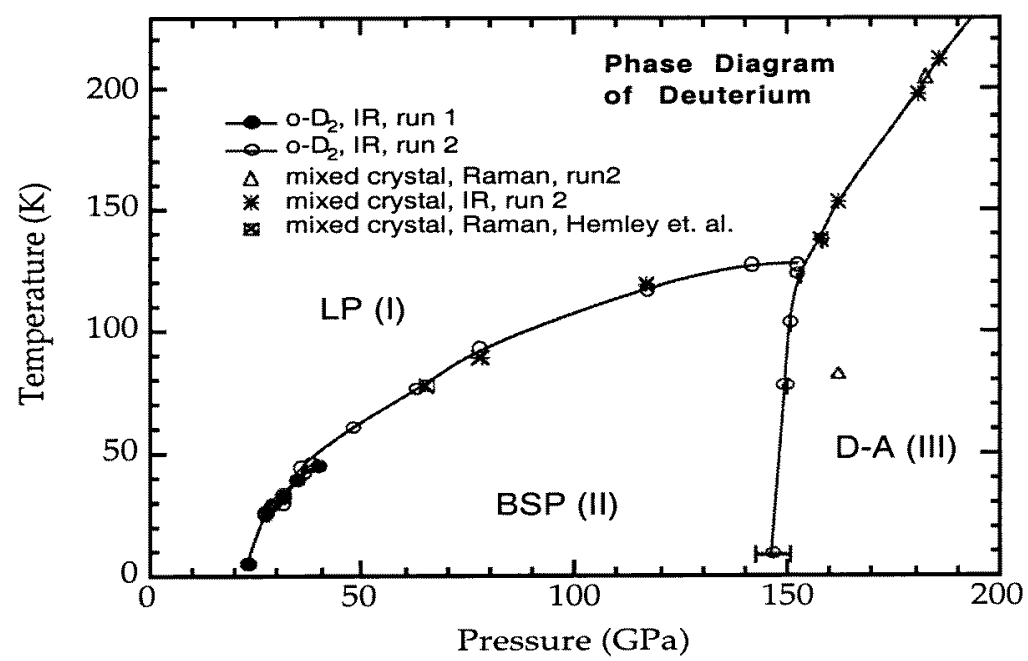

Figure 1. The phase diagram of ortho-deuterium at high pressures.

Pure ortho hydrogen at zero pressure undergoes a transition to an orientationally ordered state at $T=2.8 \mathrm{~K}$ on a face-centred cubic lattice. This is driven by the EQQ interaction which scales as $\rho^{5 / 3}$ where $\rho$ is the density. Thus, with increasing pressure (density) the transition temperature increases, as it is proportional to the strength of the anisotropic interaction. This is shown in figure 2 as the EQQ curve.

An interesting case is that of HD. Since the nucleons are distinguishable, the orientational states reach equilibrium very rapidly. The phase diagram, shown by the dots in figure 2 , has been observed [14] to be quite different from those of hydrogen and deuterium. At $T=0$ the BSP transition occurs at just under $70 \mathrm{GPa}$. If the concentration of molecules in the $J=0$ state were fixed then the phase diagram would correspond to the $c=0$ curve, where $c$ gives the concentration of molecules in the $J=1$ state. However, as temperature 


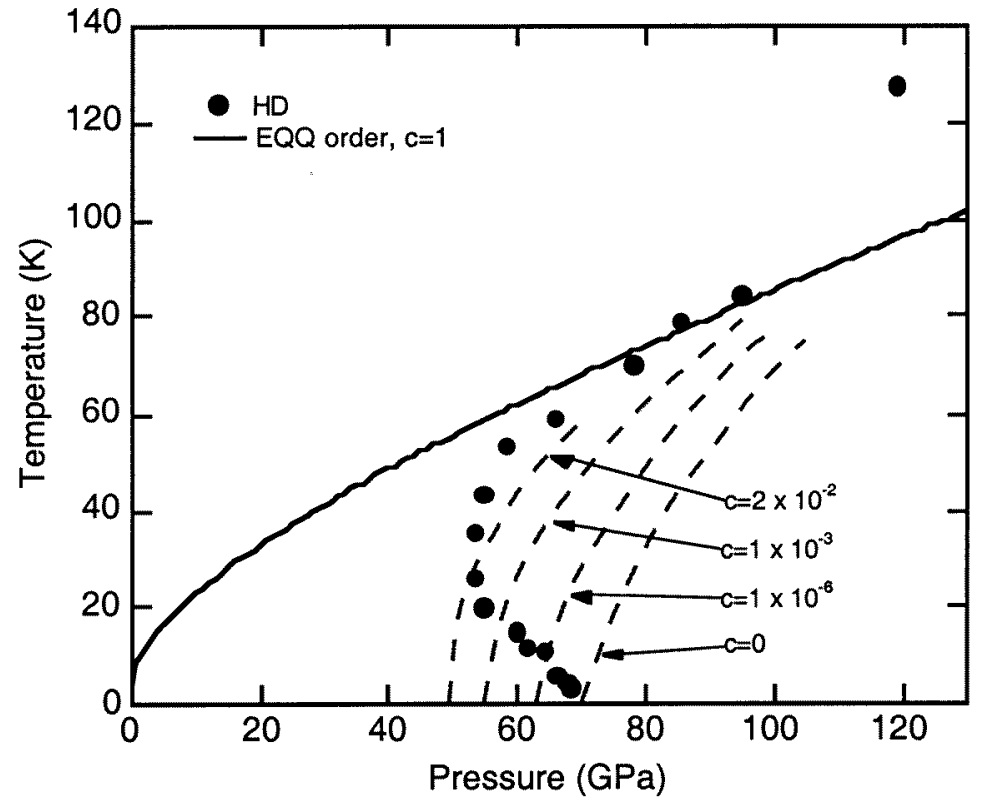

Figure 2. The phase diagram for HD showing the BSP transition (dots) and fictitious curves for samples with fixed concentration of $J=1$ species, $c$. The curve for EQQ ordering is also shown.

increases equilibrium is rapidly achieved so that the system 'jumps' the $c$ curves, resulting in the experimentally observed phase line.

\section{Intrinsic impurities: ortho and para}

Still another problem arises in hydrogen, that of intrinsic impurities. When the temperature of the lattice is changed ortho-para conversion will take place at a certain rate to achieve equilibrium. At zero pressure, this process can take weeks, while at higher pressures, days to hours. Generally a phase line is studied by holding temperature constant and traversing pressure to cross the line, or more commonly in diamond anvil cells (DACs), holding pressure constant and varying the temperature to cross the phase line. The phase transition is determined by the change in some physically measurable property such as X-ray structure, Raman or infrared spectra, etc. Now consider a lattice of pure ortho hydrogen at a pressure below one megabar and at a temperature below its ordering temperature, say at $1 \mathrm{~K}$. The equilibrium at $1 \mathrm{~K}$ is almost pure para hydrogen, so the molecules will convert towards this concentration. Since under these conditions para is orientationally 'disordered', when the ortho concentration becomes low enough, the lattice will transform to the orientationally 'disordered' para state. So a phase transition has taken place at fixed temperature and pressure just by converting towards equilibrium. From this example we can understand that a lattice of hydrogen will have a continuous number of phase diagrams, as the ortho-para concentration varies between 0 and 1 , resulting in substantial complexity in the study of hydrogen at high pressure.

Now let us consider the implications of ortho-para species for metallization. First, we have already mentioned that the orientational distributions have an important effect on the 
calculated transition pressure for metallization in the molecular system. However, there is a more important problem. If a solid is at a finite temperature then there will be a mixture of ortho and para states. The one acts as an impurity of the same mass but has different interactions from the other. This can possibly give rise to Anderson localization and suppress an insulator-metal transition that would occur in a pure lattice [15]. It might be the case that even pure ortho or para lattices suffer from Anderson localization. Since the electron motions are very much faster than the nuclear motions, an electron moving through a pure lattice sees a snapshot of molecules with random orientations, although on the average (in the quantum mechanical sense), the molecules have well defined distributions. At this time it is not clear from theoretical considerations whether the electron will sense a translationally invariant or a disordered lattice. Is it possible that the failure to observe metallization in the A-phase of hydrogen, above $150 \mathrm{GPa}$, is due to this type of disorder?

\section{Experimental efforts to observe metallic molecular hydrogen}

In the late 1980s and early 1990s there were a few claims of experimental observation of metallic hydrogen by Mao, Hemley and coworkers. First hydrogen was observed to darken above $200 \mathrm{GPa}$ [16] and this, among other things, was presented as evidence of metallization. This was refuted with arguments that darkening is not evidence of metallization, etc [17]. Next, IR reflectivity measurements in hydrogen at room temperature above $150 \mathrm{GPa}$ were presented as evidence of band overlap metallization based on comparison to a Drude model [18]. However, it was shown that these spectra were internally inconsistent, violating causality which relates the absorption to the reflection via the Kramers-Kronig relations [19]. Then the claim of metallization was forwarded again with absorption spectra and adjusted interpretation to make the reflection and absorption spectra compatible [20]. However, it was shown that the changes in the interpretation, mainly the modification of the indices of refraction of hydrogen and diamond, were unphysical [21,22]. Finally in 1996 Chen et al [23] measured the absorption spectra of hydrogen at high pressure out to 10 microns, shown in figure 3, extending the original long wavelength range of Mao and coworkers which was limited to about 2 microns. In this work it was unequivocally shown that the spectrum was not a Drude spectrum; this was also confirmed by Hemley et al [24]. The absorption in the region which was earlier claimed to be a Drude edge was due to a strong vibron peak which rises and falls in intensity, and the earlier work evidently interpreted the rising edge as a Drude edge. Chen et al studied their sample at a temperature and pressure in which it was not in the A-phase, but rather in the phase which should be the hcp phase of low pressure hydrogen. It is well known from group theoretical arguments that there should be no infrared active vibrons in the pure hcp lattice. The appearance of an absorption in the 2 micron region is attributable to a mixture of ortho and para molecules on the lattice. If the lattice were purely one species then there would be no absorption as translational invariance exists. In the impure lattice group theoretical arguments are not valid and the crystal momentum is no longer a good quantum number. The absorption was attributable to impurity spectrum of the vibron. Thus, the mixed ortho-para species was behind a long standing claim of metallization which was finally retracted in 1996 [24].

\section{The validity of ortho-para states at high pressure}

Until recently, there seems to have been a widespread misconception that the o-p states become invalid at high pressure. It has been argued that the $\mathrm{o}-\mathrm{p}$ classification breaks 


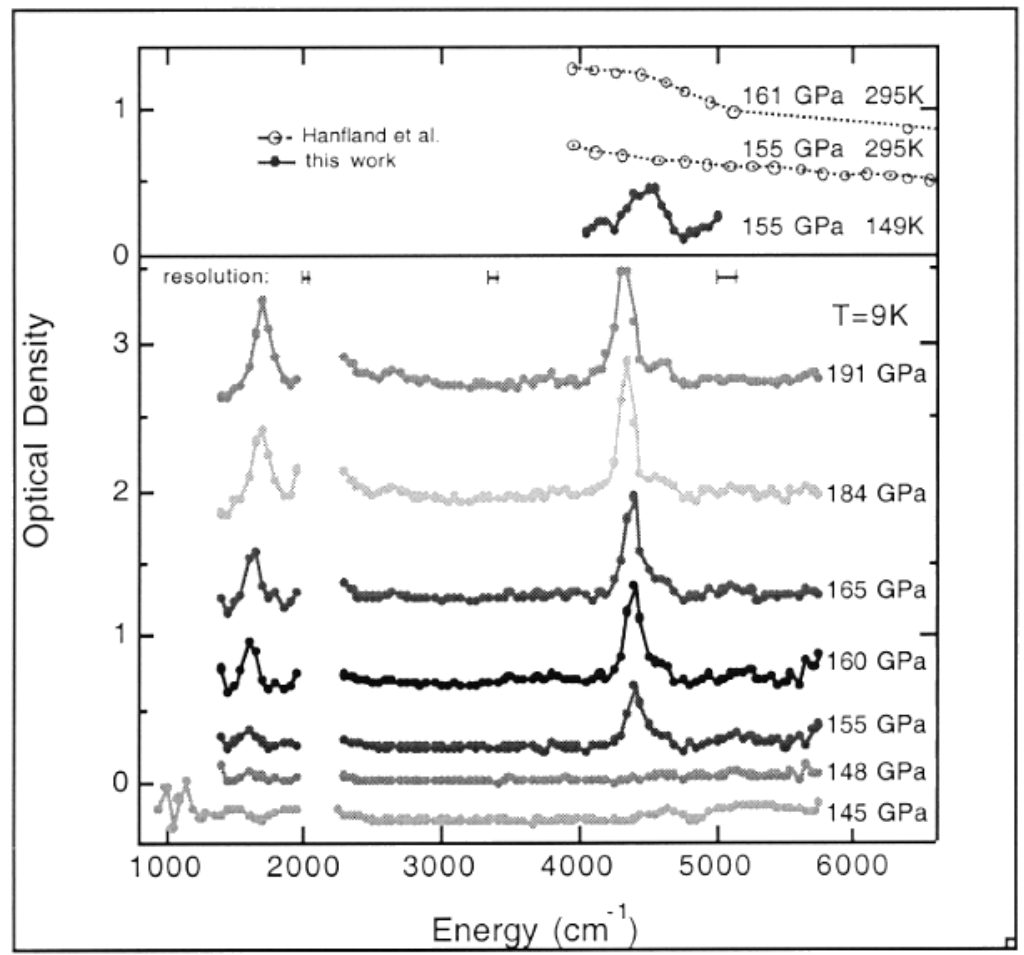

Figure 3. The near IR spectrum of hydrogen at high pressure, in the A phase, demonstrating two vibron peaks, but no Drude behaviour, characteristic of a conductor. The upper frame shows ortho impurity absorption in the LP phase.

down when the $J=0$ to 2 and 1 to 3 rotational transitions overlap; when the ortho-para conversion rate becomes sufficiently rapid; when the wavefunction of the crystal may no longer be represented as a product of molecular wavefunctions at high pressure. In a recent review article on high pressure hydrogen [25] it was stated that 'Strictly speaking, the ortho-para designation is only applicable to hydrogen where $J$ is a good quantum number and the crystal's wavefunction can be represented as a product of molecular rotational wavefunctions, as is the case at low densities'. Of course, the only important quantum number for the o-p states is parity under exchange. Thus, with increasing pressure the orientational states may change substantially, however this should not affect the orthopara concept. The nuclear spin states should be preserved until the dissociative WignerHuntington transition occurs.

In the solid, the many-body wavefunction must be antisymmetric under the exchange of protons. The most important criterion for the preservation of the ortho-para states is that the exchange of nucleons be confined to intramolecular exchange. If protons can exchange with protons on neighbouring molecules then the wavefunctions are very complicated and the concept of o-p breaks down. This has recently been discussed in detail by Silvera [26] and we mention some of the results. In the zero-pressure solid, the molecules are highly localized with little overlap and as a consequence the ortho-para concept remains valid. Detailed studies of low pressure solid helium [27] show that the exchange diminishes as the 19th power of the molar volume; a similar model has been proposed and studied for 
hydrogen [28]. We expect this approximation to maintain its validity to pressures where the Wigner-Huntington dissociative transition to atomic metallic hydrogen takes place. In this density region the intramolecular exchange approximation definitely breaks down.

The o-p concept maintains its validity as long as the molecular states are pure, that is if the wavefuntion is written as an admixture

$$
|\Psi\rangle=\left|\Psi_{\text {ortho }}\right\rangle+\alpha\left|\Psi_{\text {para }}\right\rangle
$$

then the constant $\alpha$ is very small. Silvera showed that the average value of the admixture is

$$
|\bar{\alpha}|=8 \times 10^{-10} c\left(V_{0} / V\right)^{4 / 3}
$$

where $c$ is the ortho concentration and $V$ and $V_{0}$ the molar volumes at pressure $p$ and zero pressure, respectively. Thus, even at a megabar the admixture is of the order of $10^{-8}$ to $10^{-9}$ and the o-p concept remains a very good notion.

\section{Experimental studies of ortho-para conversion}

It now seems clear that in the study of high pressure hydrogen and its isotopes it is extremely important to keep track of $\mathrm{o}-\mathrm{p}$ concentration. Some researchers have done this by maintaining their samples at low temperatures for extended periods to ensure a sample rich in para concentration, and then only holding the sample at elevated temperatures for limited periods of time, to minimize the conversion to an unknown concentration. To be able to track the condition of the sample one needs to know the conversion rate constant at a given pressure and temperature; until recently this was only known at low pressure, to about $0.6 \mathrm{GPa}$. High pressure conversion rate constants have been recently reported by Eggert et al [29] and Pravica and Silvera [30]. Eggert et al used Raman scattering to study conversion while Pravica and Silvera used NMR. Since there is little detail in optical studies we shall focus on the NMR studies.

Intrinsic o-p conversion in solid hydrogen was first studied theoretically by Motizuki and Nagamiya [31]. Conversion involves a change of nuclear spin as well as rotational state. The microscopic source for conversion of a central ortho molecule is magnetic field gradients arising from the magnetic dipolar fields of neighbouring ortho molecules. At modest temperatures the central ortho molecule is in the $|\operatorname{Im} ; J M\rangle=|1 \mathrm{~m} ; 1 M\rangle$ state and converts to the $|00 ; 00\rangle$ state. Since conversion depends on the presence of another ortho molecule, the rate equation is second order in the ortho concentration $c$,

$$
\frac{\mathrm{d} c}{\mathrm{~d} t}=-K c^{2}
$$

where $K$ is the conversion rate constant. The solution to this equation shows that $1 / c \propto K t$, so that in a plot of $1 / c$ against $t, K$ is determined from the slope. In the conversion process, energy must be conserved. The energy change is primarily the rotational energy $B J(J+1)$ which changes by $2 B / k_{B}=170.7 \mathrm{~K}$, where $B$ is the rotational constant. This energy is taken up by the lattice via phonon emission or absorption. At zero pressure the rate is quite slow as it is bottlenecked by a low density of final states. At this pressure two phonons are required to conserve energy. Berlinsky [32] has studied the theory of conversion for modest increases in pressure. Using the Motizuki-Nagamiya theory, he has found that a small pressure change increases the phonon energy so that single phonons can conserve energy, with a resulting increase in the rate constant, as observed experimentally [33-35]. However, the rate constant is expected to peak and then fall as the phonon density of states at the conversion energy decreases with rising pressure. Silvera [26] has proposed that 


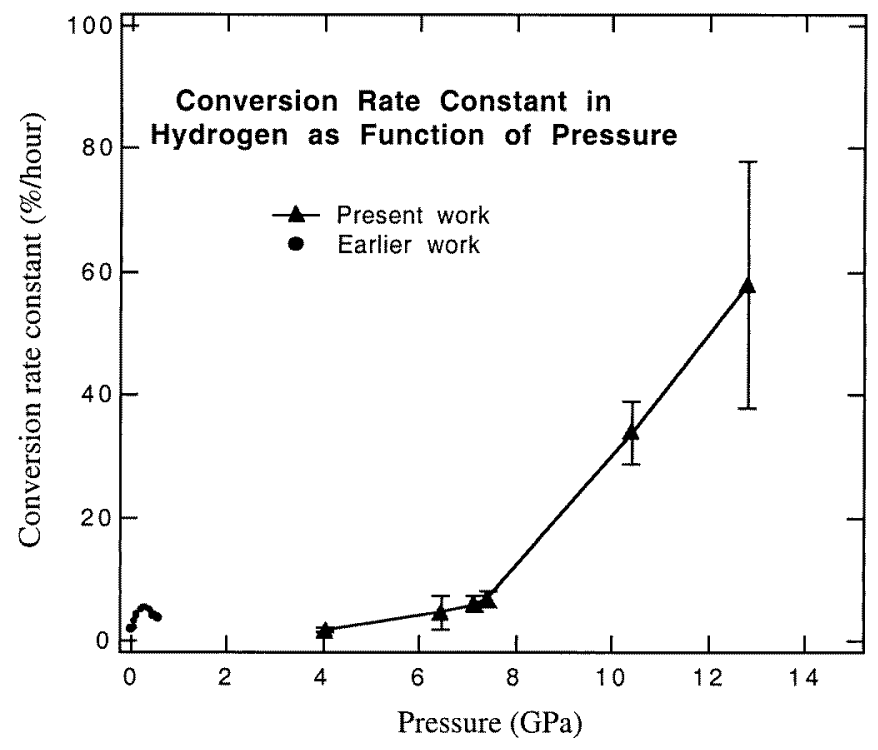

Figure 4. The conversion rate constant in hydrogen at high pressure as measured by NMR.

the rate again increases due both to stronger spin-spin coupling with higher pressure, but mainly due to the availability of rotational states which should be more strongly coupled than the phonons and take over the role of final state energy conservation in a conversion event.

To measure the o-p conversion rate constant, one notes that the NMR signal is proportional to the ortho concentration. The ortho molecules $(I=1)$ contribute to the nuclear magnetization and thus the NMR signal while the $I=0$ para molecules do not. Pravica and Silvera [36] developed a novel NMR geometry for NMR in a DAC. Earlier NMR experiments in a DAC suffered from highly attenuated signals as the metallic gaskets inhibit the RF field from penetrating into the sample. In the new geometry the gasket is slit and sealed with an insulator, so that it resembles a single loop around the sample and the full RF field penetrates perpendicular to the face of the gasket. The result is an enhancement in signal of 50-100. This can be used to reduce the size of the sample and thus achieve higher pressures.

The NMR signal in hydrogen was measured by Pravica and Silvera [30] as a function of time up to pressures of $12.8 \mathrm{GPa}$. The resulting values of $K$ as a function of pressure are shown in figure 4, along with low pressure results. At low pressure $K$ rises from about $2 \% \mathrm{~h}^{-1}$ to $6 \%$ and decreases. At the pressure where the new measurements start, $4 \mathrm{GPa}$, it is $2 \% \mathrm{~h}^{-1}$ and with increasing pressure rises rapidly to approximately $60 \% \mathrm{~h}^{-1}$ at $12.8 \mathrm{GPa}$. Clearly the rate becomes very rapid at high density and is expected to continue to increase. Eggert et al [29] have measured to even higher pressures. In the region of overlap there is good agreement with the NMR results at low pressure below $8 \mathrm{GPa}$; however, at higher pressures their rate constants are about half as large as the ones measured by NMR.

It is clear that ortho-para is an important concept. Recent experimental measurements are beginning to give quantitative values of the conversion rate constant. It will be important to extend these measurements to megabar pressures so that the o-p state of a sample can be tracked. 


\section{Conclusions}

Hydrogen has been studied at multimegabar pressures. The predicted metallization of hydrogen in the molecular phase or transition to the atomic phase is yet to be observed. Earlier claims of metallization in the solid state have been found to be groundless. The ortho-para concentration of the molecules in the solid appears to be an important aspect or property for understanding of the molecular phases at high pressure. It is not clear whether the o-p concentration will have an impact at all on the Wigner-Huntington transition to atomic metallic hydrogen.

\section{Acknowledgment}

This work was supported in part under NSF grant DMR-9701500.

\section{References}

[1] Wigner E and Huntington H B 1935 J. Chem. Phys. 3764

[2] Ashcroft N W 1968 Phys. Rev. Lett. 211748

[3] Ramaker D E, Kumar L and Harris F E 1975 Phys. Rev. Lett. 34812

[4] See for example the review by Silvera I F 1995 Metal-Insulator Transitions Revisited ed P P Edwards and C N R Rao (London: Taylor and Francis) p 21

[5] Richardson C F and Ashcroft N W 1997 Phys. Rev. Lett. 78118

[6] Chen N H and Silvera I F 1996 Rev. Sci. Instrum. 674275

[7] Chen N H 1996 PhD Thesis Harvard University

[8] Narayana C, Luo H, Orloff J and Ruoff A L 1998 Nature 39346

[9] Loubeyre P, LeToullec R, Hausermann D, Hanfland M, Hemley R J, Mao H K and Finger L W 1996 Nature 383702

[10] Ashcroft N W 1990 Phys. Rev. B 4110963

[11] Garcia A, Barbee T W, Cohen M L and Silvera I F 1990 Europhys. Lett. 13355

[12] Lorenzana H E, Silvera I F and Goettel K A 1989 Phys. Rev. Lett. 632080

[13] Lorenzana H E, Silvera I F and Goettel K A 1990 Phys. Rev. Lett. 641939

[14] Moshary F, Chen N H and Silvera I F 1993 Phys. Rev. Lett. 713814

[15] Ashcroft N W 1993 J. Non-Cryst. Solids 156621

[16] Mao H K and Hemley R J 1989 Science 2441462

[17] Silvera I F 1990 Science 247863

[18] Mao H K, Hemley R J and Hanfland M 1990 Phys. Rev. Lett. 65484

[19] Eggert J H, Moshary F, Evans W J, Lorenzana H E, Goettel K A, Silvera I F and Moss W C 1991 Phys. Rev. Lett. 66193

[20] Hanfland M, Hemley R J and Mao H K 1991 Phys. Rev. B 438767

[21] Silvera I F 1991 Proc. NATO ARW on Frontiers of High Pressure Research ed H D Hochheimer and R D Etters (New York: Plenum) p 101

[22] Ruoff A L and Ghandehari K 1993 Mod. Phys. Lett. B 7907

[23] Chen N H, Sterer E and Silvera I F 1996 Phys. Rev. Lett. 761663

[24] Hemley R J, Mao H-K, Goncharov A F, Hanfland M and Struzhkin V 1996 Phys. Rev. Lett. 761667

[25] Mao H and Hemley R J 1994 Rev. Mod. Phys. 66671

[26] Silvera I F 1998 J. Low Temp. Phys. 112237

[27] Delrieu J M and Sullivan N S 1981 Phys. Rev. B 233197

[28] Kisvarsanyi E G and Sullivan N S 1995 Phys. Rev. B 513462

[29] Eggert J H, Karmon E, Mao H K and Hemley R J 1996 Bull. Am. Phys. Soc. 41615

[30] Pravica M G and Silvera I F 1998 submitted

[31] Motizuki K and Nagamiya T 1956 J. Phys. Soc. Japan 1193

[32] Berlinsky A J 1975 Phys. Rev. B 121482

[33] Ahlers G 1964 J. Chem. Phys. 403123

[34] Pedroni P, Meyer H, Weinhaus F and Haase D 1974 Solid State Commun. 14279

[35] Driessen A, van der Poll E and Silvera I F 1984 Phys. Rev. B 302517

[36] Pravica M G and Silvera I F 1998 Rev. Sci. Instrum. 69479 